\title{
Speed Estimation in Forward Scattering Radar by Using Standard Deviation Method
}

\author{
Mutaz Salah, MFA Rasid \& RSA Raja Abdullah \\ Department of Computer and Communication Engineering \\ Universiti Putra Malaysia (UPM) \\ 43400 Serdang, Selangor, Malaysia \\ Tel: $60-3-8946-4347$ \\ E-mail: rsa@eng.upm.edu.my,fadlee@eng.upm.edu.my,mutazoya@hotmail.com
}

\author{
M Cherniakov \\ School of Electronic, Electrical \& ComputerEngineering \\ The University of Birmingham \\ Edgbaston, United Kingdom
}

\begin{abstract}
Forward Scattering Radar (FSR) is a special type of Radar. The interest in FSR is rises after its capability in target classification is validated. Unfortunately, the speed of the target still not been solved. Thus, this paper proposed a method to estimate speed FSR system. The theory of FSR systems is briefly described together with practical experiments to evaluate the feasibility of such a system in real-life scenarios. All data are collected from the practical experimentation by using typical ground vehicle as target (e.g. car, lorry). In the proposed system, Standard Deviation for each raw radar signal is calculated. This Standard Deviation information is exploited to estimate the speed of a target. This method shows a good result in estimating the vehicle speed crossing FSR baseline. By analyzing 850 experimentally obtained car signatures, the performance of the system is evaluated and the effectiveness of the system is confirmed.
\end{abstract}

Keywords: Forward scattering radar, Standard Deviation method and speed estimation

\section{Introduction}

There are different types of radar systems based on the transmitter-receiver topology as shown in Figure 1. Forward scattering radar (FSR) is a special mode of bistatic radar that can be used for target detection and classification. FSR offer a number of interesting features such as: relatively simple hardware (Gould, D.M., Orton, R.S., Pollard, R.J.E., 2002)( Willis N. J., 1995); an enhanced target radar cross section (compared to traditional radar)(Chernyak, 1998); a long coherent interval of the receiving signal; robustness to stealth technology and possible operation using non-cooperative transmitters. Recent research has shown its capability in target classification (M. Cherniakov, R.S.A.R. Abdullah, P. Janc ̌ovič, M. Salous and V. Chapursky, 2006)( Abdullah R., Cherniakov M., Jancovic P. 2004)( M Cherniakov, Salous, Kostylev and RSA Abdullah. 2005)( M Cherniakov, V.V Chapurskiy, RSA Raja Abdullah, P.Jancovic, M. Salous. 2004)( RSA Raja Abdullah, M Cherniakov, 2003). Although FSR has an advantage compared to the traditional radar, but it also has number of known drawbacks, such as the absence of range resolution and narrow operational area, etc.

In references (M. Cherniakov, R.S.A.R. Abdullah, P. Jančovič, M. Salous and V. Chapursky, 2006) which describe the automatic target detection using FSR, the speed is estimated by analyzing the information from the video camera. Thus, this paper is filling the gap towards the current research in FSR by estimating the speed of a target. The information from the Standard deviation of a raw radar signal is analyzed to predict the speed of a target crossing the FSR baseline. It is shown that using these techniques the speed can be effectively estimated. This paper is dedicated to the experimental study of the feasibility of FSR and its' application for target (ground vehicle) speed estimation. It introduces the radar system itself, fundamental theoretical analysis, and the targets speed in relation to Doppler frequency. By using FSR for detection and classification of ground targets together with the low cost system, it could find various applications in civil, defense, medical and security systems. 


\section{Forward Scattering Radar basics}

Before the speed estimation procedure is described in detail, this section summarizes the basic technical of FSR in terms of signal scattered and power budget. More detail about this subject can be found in (M. Cherniakov, R.S.A.R. Abdullah, P. Janc ovič, M. Salous and V. Chapursky, 2006) for FSR theoretical background and in (Sizov, V; Cherniakov, M.; Antoniou, M, 2007) for power budget analysis. References(M. Cherniakov, R.S.A.R. Abdullah, P. Jančovič, M. Salous and V. Chapursky, 2006)( Abdullah R., Cherniakov M., Jancovic P. 2004)( M Cherniakov, Salous, Kostylev and RSA Abdullah. 2005)( M Cherniakov, V.V Chapurskiy, RSA Raja Abdullah, P.Jancovic, M. Salous. 2004)( RSA Raja Abdullah, M Cherniakov, 2003). (Blackman, A.B., Ryndyk, A.G., and Sidorov, S.B. 2000)( Chapurskiy V.V, Sablin V.N. 2000) have elaborate on the basic FSR system and it is shown again in Figure 2. It is comprises of a transmitter, Tx with $f_{c}$ central frequency with an appropriate wavelength, $\lambda$ and a receiver, $\mathrm{Rx}$ separated by a distance, $b$ from the transmitter. The target is assumed to be moving along a trajectory that crosses the baseline with speed, $V$ and has zero elevation as the system operates in a ground plane. For a moving target, the received signal experiences Doppler shift, $f_{d b r}$ and can be evaluated as (M. Cherniakov, R.S.A.R. Abdullah, P. Janc `ovič ${ }^{2}$ M. Salous and V. Chapursky, 2006):

$$
f_{d b r}=2 V \frac{1}{\lambda} \cos \delta \cos (\beta / 2)
$$

where $\beta$ is the bistatic angle.

The maximum Doppler shift occurs for the target trajectory normal (perpendicular) to the baseline. Even in this case, the absolute value of $d b r$ is essentially less than its maximal rate fdmax $=2 \mathrm{~V} / \lambda$ corresponding to a monostatic radar. The information from the scattered Doppler frequency is used in further processing for target classification from the assumption that a unique target possesses a unique Doppler signature in frequency domain (M. Cherniakov, R.S.A.R. Abdullah, P. Jančovič, M. Salous and V. Chapursky, 2006). The Doppler shift depends mainly on the target velocity vector components and the carrier frequency. The general equation that describes the received waveform, that is, target signature from the moving sample target with a rectangular shape is described in (RSA Raja Abdullah, M Cherniakov, 2003).

It is expected that the boundary for FSR is around $\beta \sim 170^{\circ}$. At the time when the bistatic angle $\beta$ approaches to $180^{\circ}$, the target blocks part of the transmitted signal, which leads to a reduction of the received signal power. In this case, the target acts as an aperture antenna with a maximum gain as (RSA Raja Abdullah, M Cherniakov, 2003):

$$
G=\frac{4 \pi A}{\lambda^{2}}
$$

where the signal wavelength, $\lambda$ is assumed to be small compared to the target's dimensions, and $\boldsymbol{A}$ is the target shadow silhouette area projection on the transmitter-target line. When $\beta=180^{\circ}$, the level of received signal reaches its maximum and the target can be characterized by a forward scattering cross-section (FSCS), $\sigma_{b}$, which also depends upon the target shadow silhouette area $A$ (RSA Raja Abdullah, M Cherniakov, 2003):

$$
\sigma_{b}=\frac{4 \pi A^{2}}{\lambda^{2}}
$$

The increase in FSCS inherently contributes to the total received signal in the power budget equation (RSA Raja Abdullah, M Cherniakov, 2003).

The reason why the speed information is silence to FSR is illustrated in the Figure 3. Let assume that the transmit time for the two positions of target be $T_{o}=0 \mathrm{sec}$. The received signal scattered by the target arrive at the receiver at the same time regardless of the target position on the FRS baseline, hence the range resolution which is critical parameter to measure speed does not exist in the received signal.

\section{Vehicle data collection and speed estimation}

An experiment to collect data using real vehicles was carried out on a public road using the setup shown in Figure 4 (M Cherniakov, V.V Chapurskiy, RSA Raja Abdullah, P.Jancovic, M. Salous. 2004). During this experiment, signals from a random stream of vehicles, as well as a number of test vehicles were collected and recorded. A video recorded the vehicles passing through the beam and allowed the speed with which the vehicles passed through the beam to be calculated. Figure 4 also shows the positioning of the video camera and a typical video scene. Two posts were attached to a tree and a lamppost and have been highlighted in the scene. The two posts were $16 \mathrm{~m}$ apart. By playing back the recorded video, the speed of a particular vehicle can be evaluated by finding the time taking for the vehicle to travel between the two posts, so we can compare the result of vehicle speed after applying the proposed method with the result from video camera. The Figure 5 shows the example of received signal in time domain from the experiment for two different vehicles crossing the FSR baseline. 
Our aim is to find the vehicle speed when the vehicles cross the sensor or the forward scattering radar system. However, speed information, in general, is not available from the radar signal itself (assuming the length of vehicle is unknown). Therefore, it is necessary to develop a practical algorithm for speed estimation. This section describes the algorithm used during the experimentation.

Vehicle speed was measured using the relationship between the time-domain signature and the speed of the vehicle as estimated from the captured video footage. In order to establish this relationship, the speed of vehicle was associated with the value of standard deviation that calculated to each vehicle signature. Figure 6 shows the example of received signal in time domain for different speeds. The graphs show that as vehicle speed increased, the vehicle's time-domain signature is compressed and vice versa. This indicates that a relationship exists between Standard deviation values for the time domain signal and the speed of vehicle. Thus, Figure 7 illustrates the general block diagram of the proposed method which utilizing the value of Standard Deviation as a steps in estimating the speed of vehicle crossing the FSR sensor.

\subsection{Standard Deviation}

In probability and statistic, the standard deviation, $\sigma$ (STD)of a probability distribution is a measure of the spread of its values. It is defined as the square root of the variance which is the average of the squared differences between data points and the mean. STD, $\sigma$ being the square root of that quantity therefore measures the spread of data about the mean (Berger J.O. 1985). In this paper, a default Matlab code is used to find the STD values for the time-domain signals. The matlab code is denoted by $\mathrm{S}=\mathrm{Std}(\mathrm{X})$, where $\mathrm{X}$ is the time-domain signal and it returns the STD by using equation (4):

$$
s=\left(\frac{1}{n-1} \sum_{i=1}^{n}\left(x_{i}-\bar{x}\right)\right)^{1 / 2}
$$

Where

$$
\bar{x}=\frac{1}{n} \sum_{i=1}^{n} \underset{i}{x}
$$

$n$ is the number of elements in the sample, $\bar{x}$ is the mean average and $x$ is the random variables. The steps to find the STD to the time-domain vehicle signature are shown below:

i. Compute the mean for the data set.

ii. Compute the deviation by subtracting the mean from each value.

iii. Square each individual deviation.

iv. Add up the squared deviations.

v. Divide by one less than the sample size.

vi. Take the square root.

\subsection{Relationship between the speed of vehicle and the standard deviation}

The assumption to associate the standard deviation value to the vehicle speed is described in this section. The basis of assumption is depending on the Doppler effect which can be described as the change in frequency when either the receiver or the energy source are in motion relative to each other. In the considered case, the source of the scatter field is moving relative to the receiver due to the motion of the vehicle; the frequency of the received signal will be shifted due to the Doppler effect. As the vehicle, in our case, is considered to be moving perpendicular to the baseline, the Doppler shift will vary throughout the vehicle's motion. Figure 8 illustrates the overall FSR system layout as seen from above and at the scene during experimentation.

When the vehicle enters the sensor coverage area from one side (position ' $a$ ' in Figure 10), the Doppler shift will be large, reducing to zero as the vehicle reaches the baseline (position ' $b$ '). The Doppler shift then increases again as the vehicle moves towards the other side of the coverage area (position 'c'). So, in theory, the Doppler frequency is larger as the vehicle enters and leaves the sensor coverage area, and the Doppler frequency is zero when the vehicle is on the baseline. Figure 9 shows the variation of the Doppler frequency relative to the scattering point of the vehicle

The general equation that describes the received waveform, i.e. the target signature from the moving sample target is explained in detail in (RSA Raja Abdullah, M Cherniakov, 2003) and it is summarised below:

$$
V_{y} \approx \Re \sin \left(\frac{4 \pi v \sin (\alpha)}{\lambda} t\right)
$$


where

$$
\Re=K_{A} \frac{l h E_{o} \cos ^{2}(\alpha)}{2 \lambda z_{b}} \frac{\sin \left(\frac{\pi l \cos (\alpha)}{\lambda} \alpha\right)}{\frac{\pi l \cos (\alpha)}{\lambda} \alpha}
$$

$l$ and $h$ are the target's length and height respectively, $\Re$ is the amplitude of the Doppler components, $v$ is the velocity of the sample target passing through the sensor, $K_{A}$ is the receiving antenna transformation coefficient, $z_{b}$ is the distance between the target crossing point on the baseline to the receiver and $E_{o}$ is a constant. This equation was derived for a rectangular shaped target. The Doppler frequency can be extracted by the derivation of the phase component of equation (7) and given by (RSA Raja Abdullah, M Cherniakov, 2003):

$$
f_{d}=\frac{2 v}{\lambda} \sin \alpha
$$

From equation (8) it is obvious that the Doppler shift depends mainly on the target velocity vector components and the carrier frequency. This Doppler shift can be used for moving target selection (M. Cherniakov, R.S.A.R. Abdullah, P.

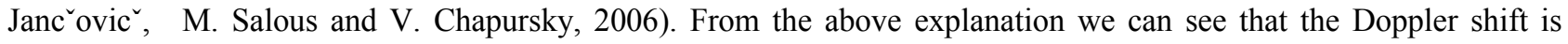
related to the speed of vehicle and this also guide us to find the relation between the standard deviation value and the speed of vehicle because whenever the speed is changing, the value of standard deviation is also changing as shown in Figure 10 for different speeds.

\section{Automatic speed estimation structure in FSR}

The overall automatic system is divided into two phases: the first phase (known as training phase) is to create a database (Speed vs. Standard deviation) which will generate the final relationship of the two parameters. Here, the information from the video camera is needed as a reference. The next phase is where the speed determination is processed, and it is known as the testing phase. In this phase, the unknown speed is compared with the database which was generated from phase one. Figure 11 illustrates the overall structure

In total, 700 samples for different vehicle with different speeds were used in the training phase and are plotted in Figure 12. This plot (database) will indicate the correlation between the speed and Standard deviation value and for this case a linear relation was generated. A line of 'best fit' was determined for this plot and it is used to find the unknown vehicle speed during testing phase. The line of best fit give the relationship between the value of standard deviation and the speed of the vehicles and for this plot is given by:

$$
\text { Vehicle speed }=20 * \text { Standard Deviation Value }-1.5
$$

In the testing phase, the Standard deviation for unknown time domain signal input is calculated in the pre-processing stage. The standard deviation value is then substitute into the equation (9) to find the speed. A total of 150 samples were used in testing phase and Figure 13 shows the plot of using equation (9).

To show that the length of vehicle is not related to the standard deviation, a plot of 30 samples of different vehicle length but with same speed $8.1 \mathrm{~m} / \mathrm{s}$ as illustrated in Figure 14. The graph shows that the standard deviation values stay the same for different value of vehicle length. To analyze the reliability of the proposed method, this section investigates the Root Mean Square (RMS) Error between the speed estimated from the algorithm and from the video camera. The normalized RMS error is calculated for this data using equation (10) (Pawel L., Thomas H. 2005).

$$
\text { Error }=\frac{\delta}{\text { mean }}=\frac{\left\{\frac{1}{n-1} \sum_{i=1}^{n}\left(x_{i}-\bar{x}\right)^{2}\right\}^{\frac{1}{2}}}{\bar{x}}
$$

where $\mathrm{n}$ is the number of data, $\sigma$ is standard deviation and $\bar{x}=\frac{1}{n} \sum_{i=1}^{n} x_{i}$.

For the plot in Figure 14 the RMS error is $22 \%$. This error is acceptable in FSR application especially for classification using FSR system.

\section{Conclusion}

This paper presents vehicle speed estimation in special mode of radar system known as forward scattering radar. Standard deviation characteristics are utilized to achieve the objective. The value of Standard deviation for each signal is unique for different speed. Thus, it carries information about the speed of the vehicle. This is confirmed by data obtained from practical experiments with a system that was developed in-house. The proposed method shows a better 
accuracy compared to the previous method first introduced in (Blackman, A.B., Ryndyk, A.G., and Sidorov, S.B. 2000). This paper also shown that, more hidden information can be possibly extracted from the FSR signal with signal processing techniques.

\section{References}

Abdullah R., Cherniakov M., Jancovic P.(2004).Automatic Vehicle Classification in Forward Scattering Radar, First International Workshop on Intelligent Transportation WIT Hamburg, Germany, Proceedings pp. 7-12.

Berger J.O. (1985).’'Statistical Decision Theory and Bayesian Analysis.” Springer-Verlag 2 ${ }^{\text {nd }}$, ISBN 3540960988

Blackman, A.B., Ryndyk, A.G., and Sidorov, S.B.(2000).Forward Scattering Radar Moving Object Coordinate Measurement". Int.Radar Conf.,Washington, DC, USA, pp. 678-682.

Chapurskiy V.V, Sablin V.N.(2000).SISAR: Shadow Inverse Synthetic Aperture Radiolocation, International Radar Conference, The Record of the IEEE 2000 International, pp. $322-328$.

Chernyak.(1998). Fundamentals of multisite radar systems. Gordon and Breach Science Publishers.

Gould, D.M., Orton, R.S., Pollard, R.J.E. (2002). Forward Scatter Radar detection, RADAR 2002, 15-17 , pp. 36 - 40.

M Cherniakov, Salous, Kostylev and RSA Abdullah. (2005).Analysis of Forward Scattering Radar for Ground Target Detection. European Radar Conference, pp.145 - 148.

M Cherniakov, V.V Chapurskiy, RSA Raja Abdullah, P.Jancovic, M. Salous.(2004).Short- Range Forward Scattering Radar, International Radar Conference, The Record of the IEEE International, pp. 322 - 328.

M. Cherniakov, R.S.A.R. Abdullah, P. Janc`ovic``, M. Salous and V. Chapursky (2006) Automatic ground target classification using forward scattering radar, IEE Proc.- Radar Sonar Navig., Vol. 153, No. 5,

Pawel L., Thomas H. (2005). “Statistics Methods and Applications”, Statsoft Inc, ISBN 1884233597.

RSA Raja Abdullah, M Cherniakov (2003).Forward Scattering Radar For Vehicles Classification ,VehCom International Conference, VehCom2003, UK, pp.73-78.

Sizov, V; Cherniakov, M.; Antoniou, M (2007).'Forward Scattering Radar Power Budget Analysis For Ground Targets. Radar, Sonar \& Navigation, IET, 1, Issue 6, pp437 - 446

Willis N. J. (1995) Bistatic Radar Technology Service Corporation.

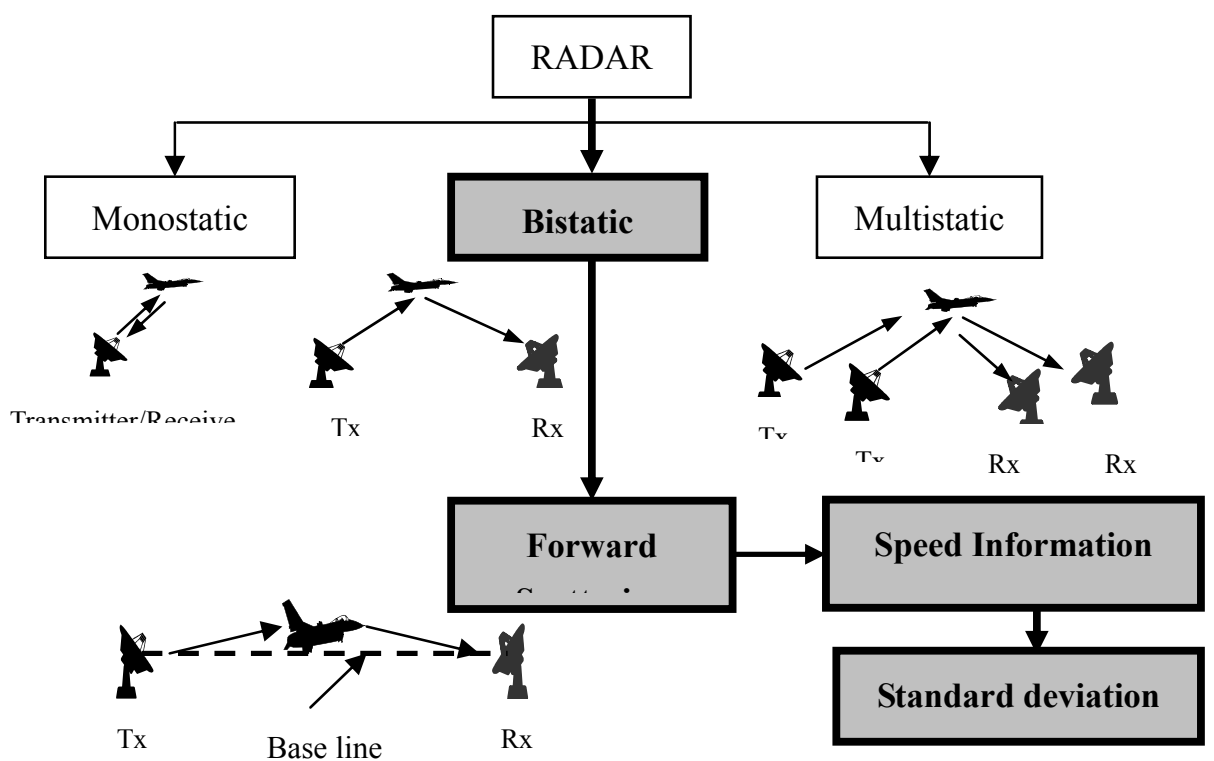

Figure 1. Radar System Classification 


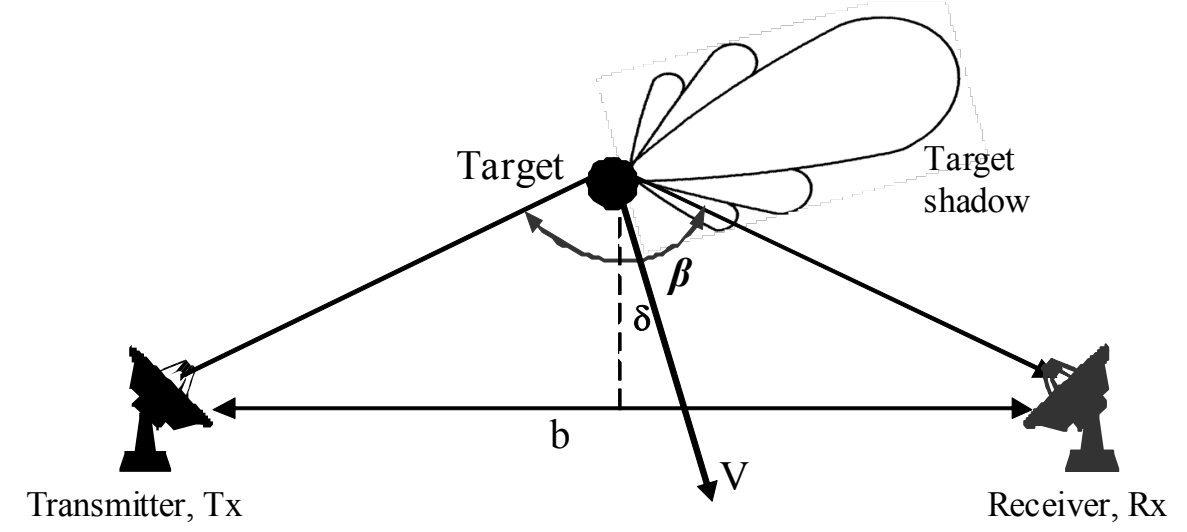

Figure 2. FSR general layout with target trajectory

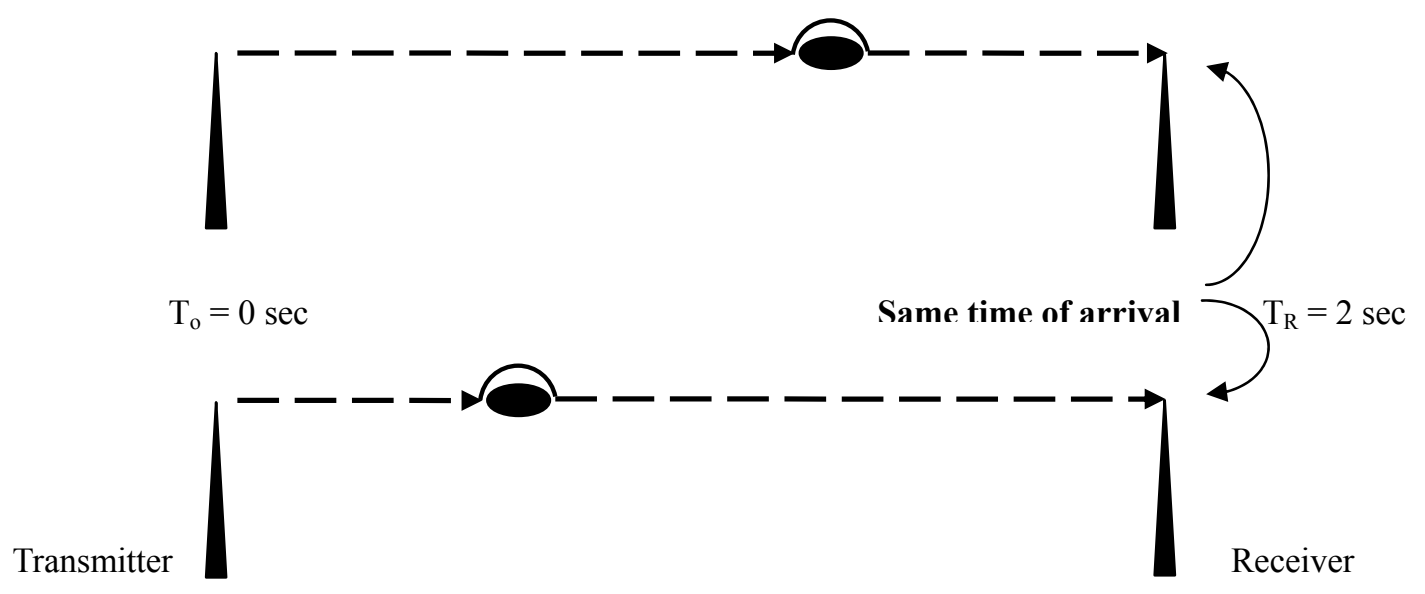

Figure 3. Causes of loss in range resolution

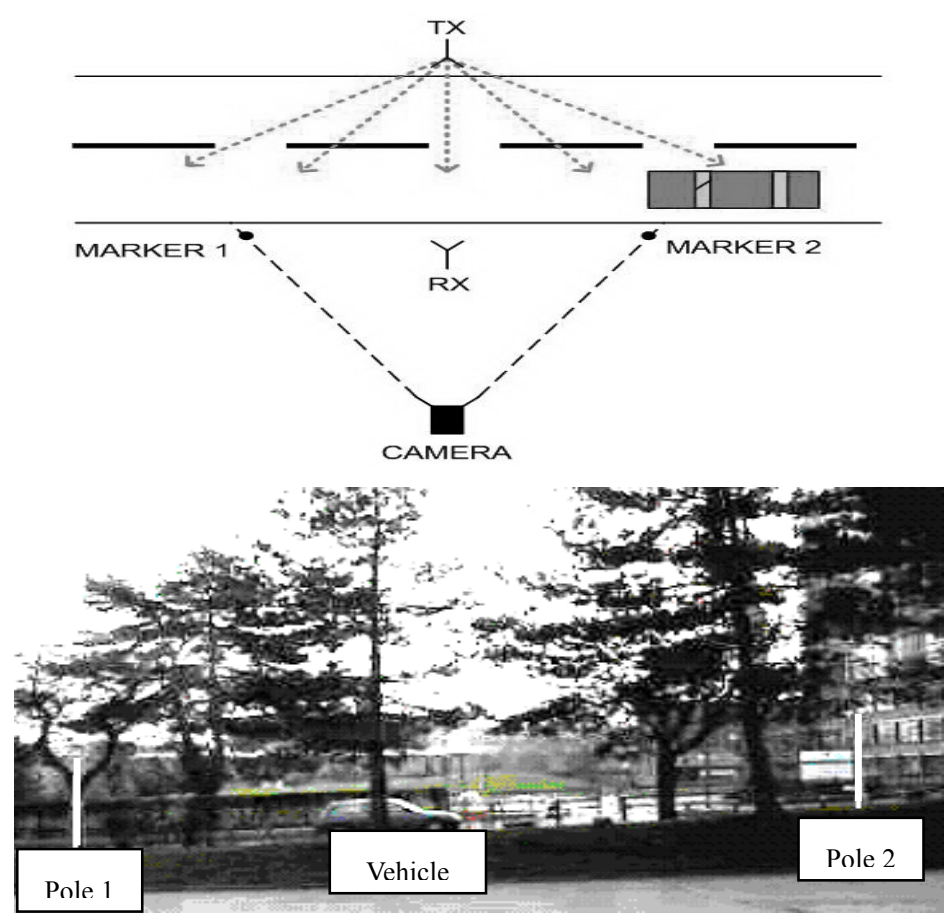

Figure 4. Experimental setup block diagram and typical video from the test day 

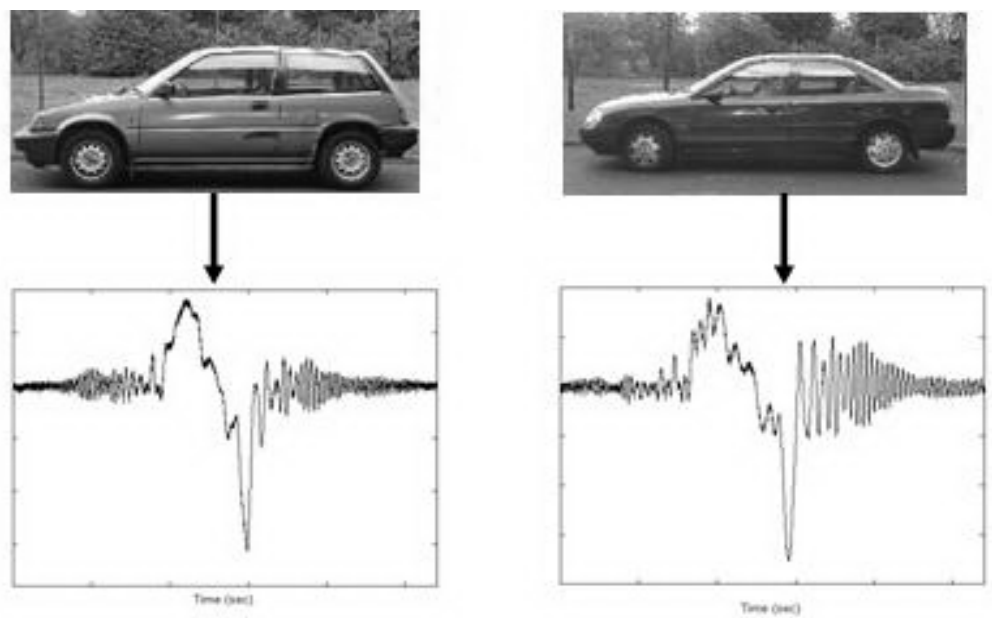

Figure 5. Time domain signals for different vehicle

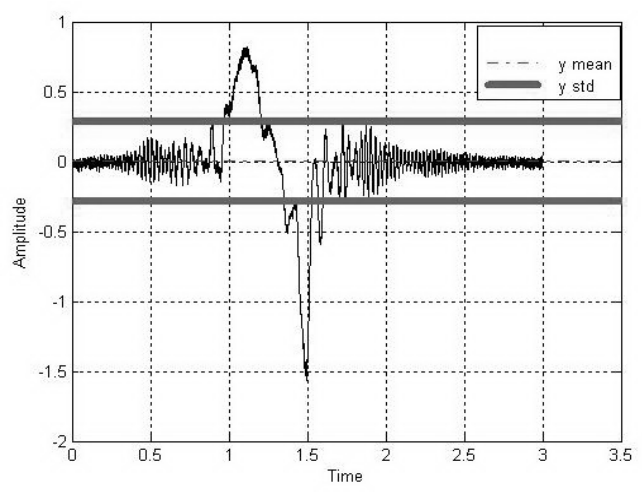

Speed $=3.61 \mathrm{~m} / \mathrm{s}, \mathrm{Std}=0.25$

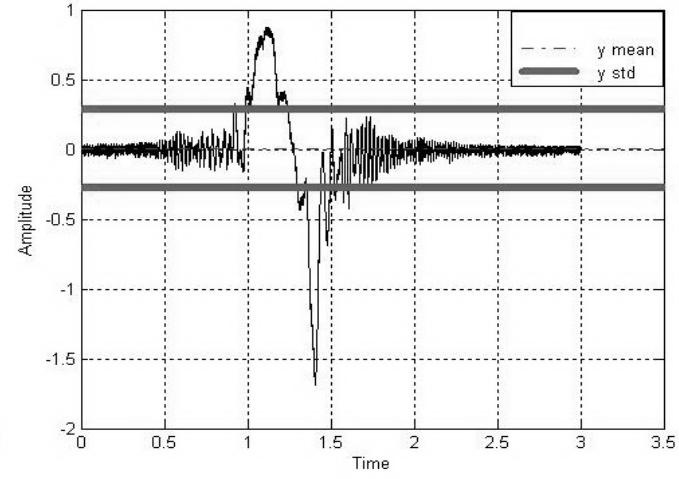

Speed $=11.11 \mathrm{~m} / \mathrm{s}, \quad$ Std $=0.6$

Figure 6. Different speed signature and its Standard Deviation

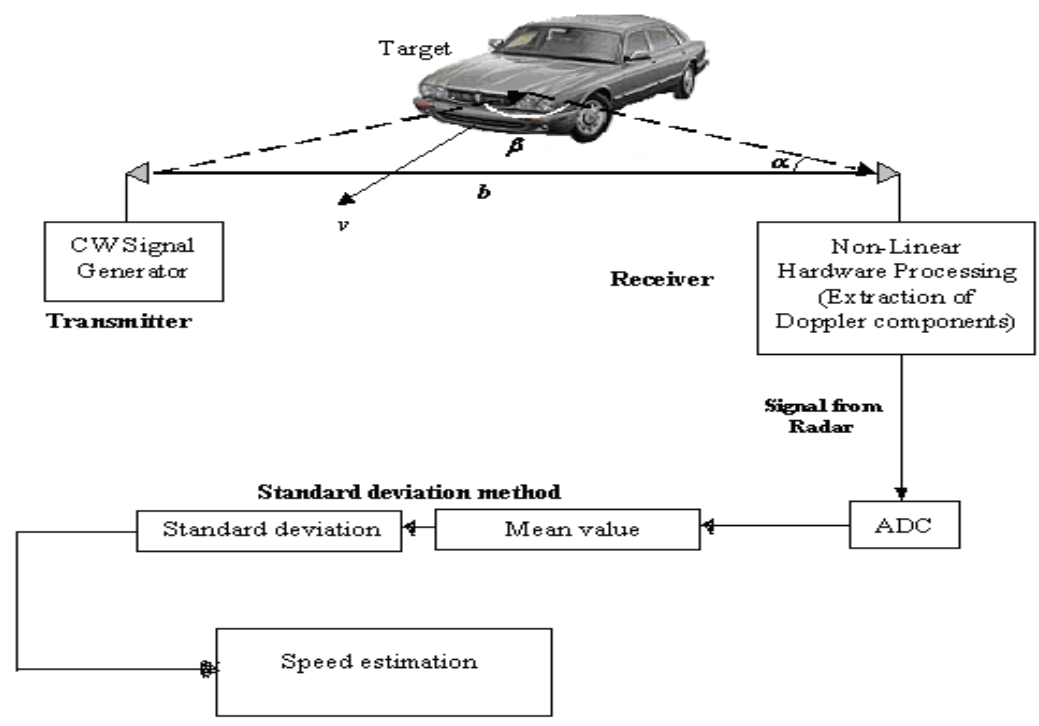

Figure 7. General block diagram for speed estimation in FSR system 


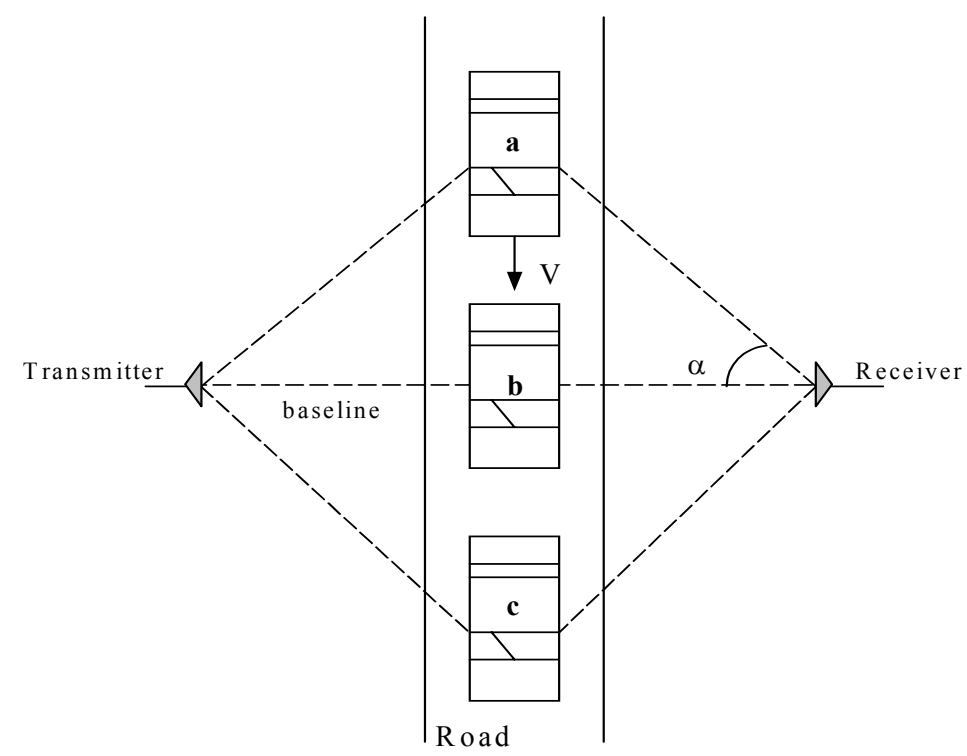

Figure 8. Overall FSR system layout from above ( $\mathrm{a}, \mathrm{b}$ and $\mathrm{c}$ are the vehicle positions)

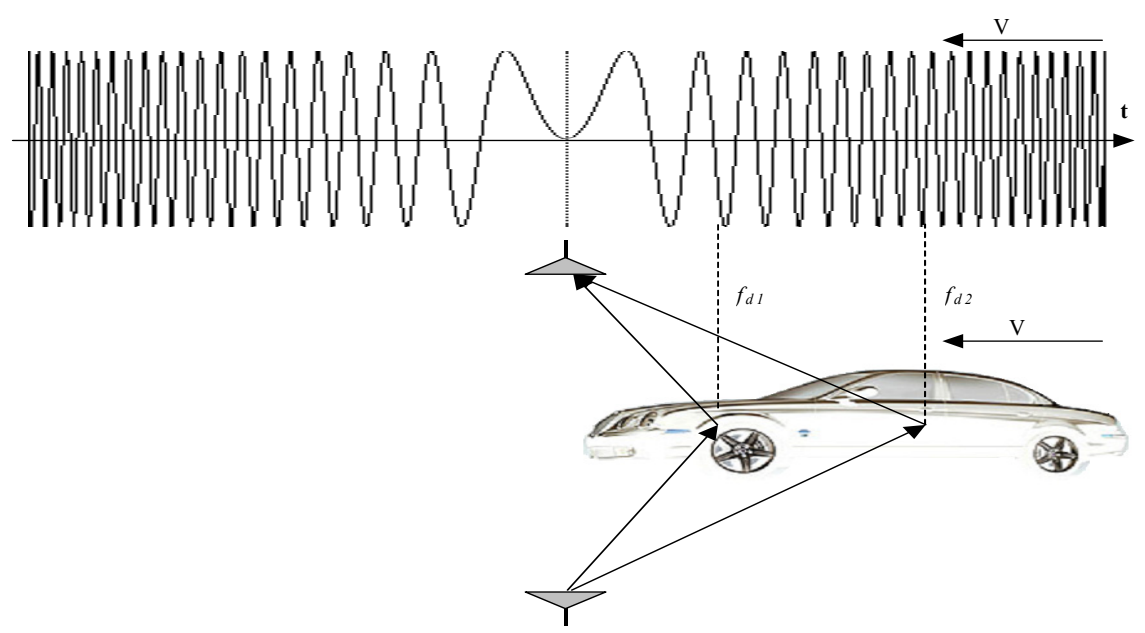

Figure 9. Doppler frequency variation relative to the scattering point on the vehicle

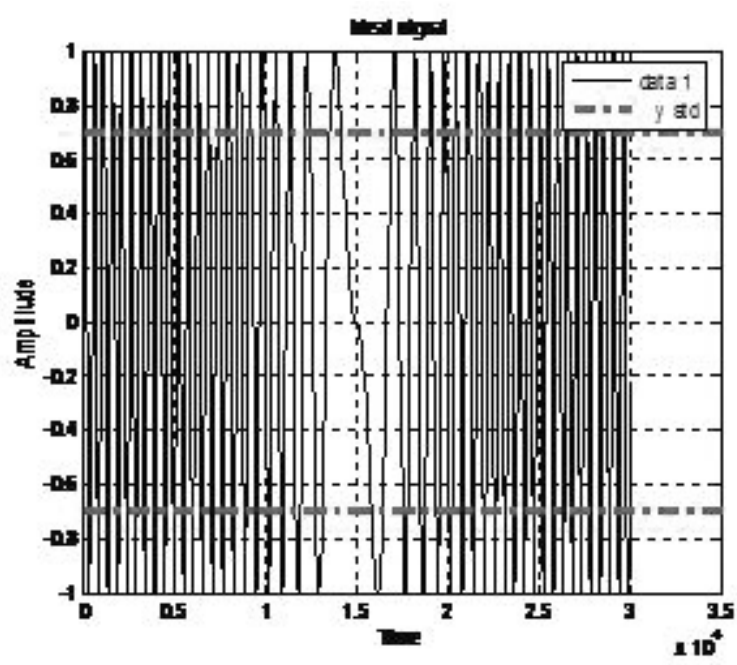

Speed $=7 \mathrm{~m} / \mathrm{s}, \mathrm{STD}=0.201$ 


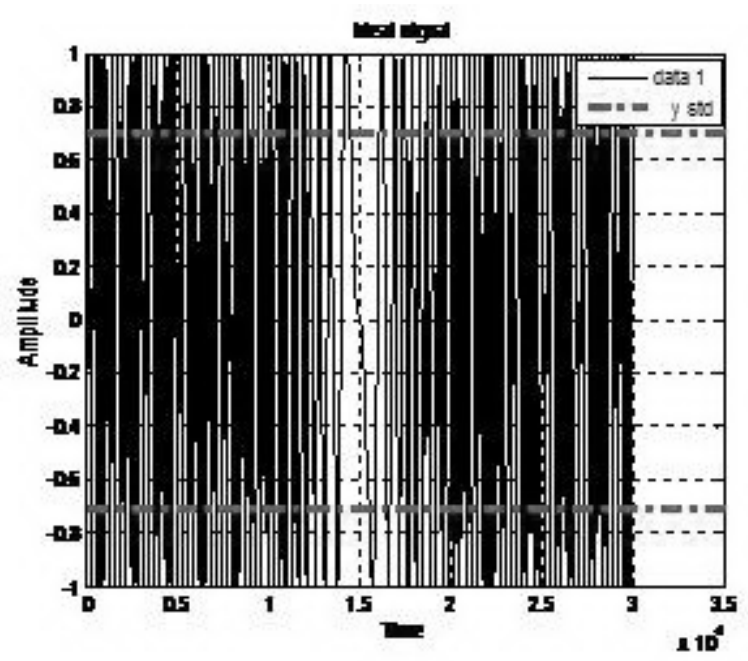

Speed $=12 \mathrm{~m} / \mathrm{s}, \mathrm{STD}=0.251$

Figure 10. Theoretical Doppler signal for different speed for target with rectangular shape

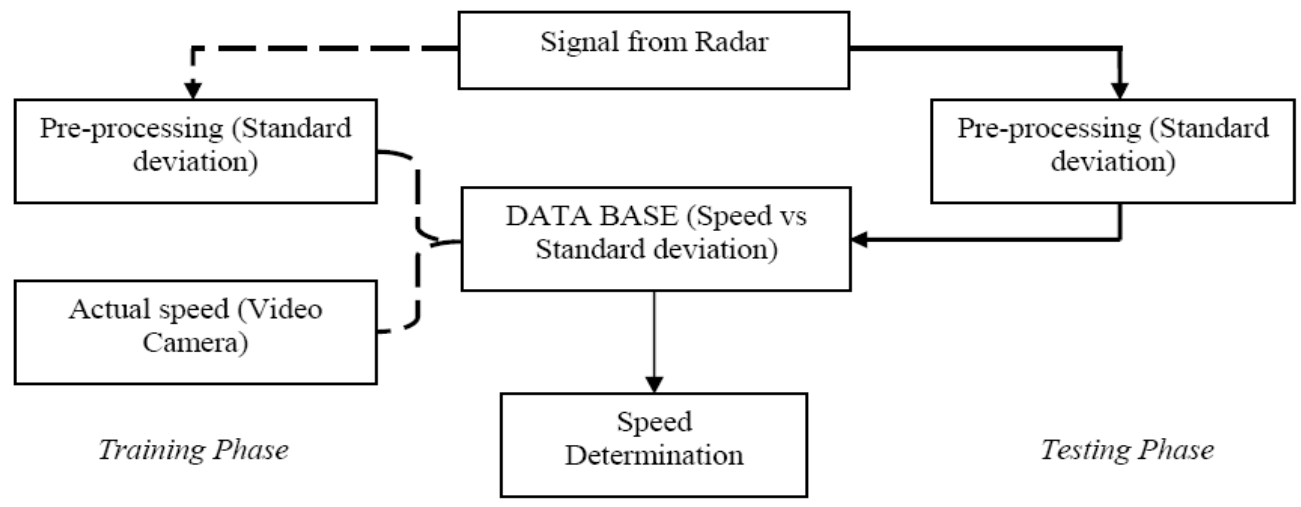

Figure 11. Block diagram for Speed Estimation in FSR

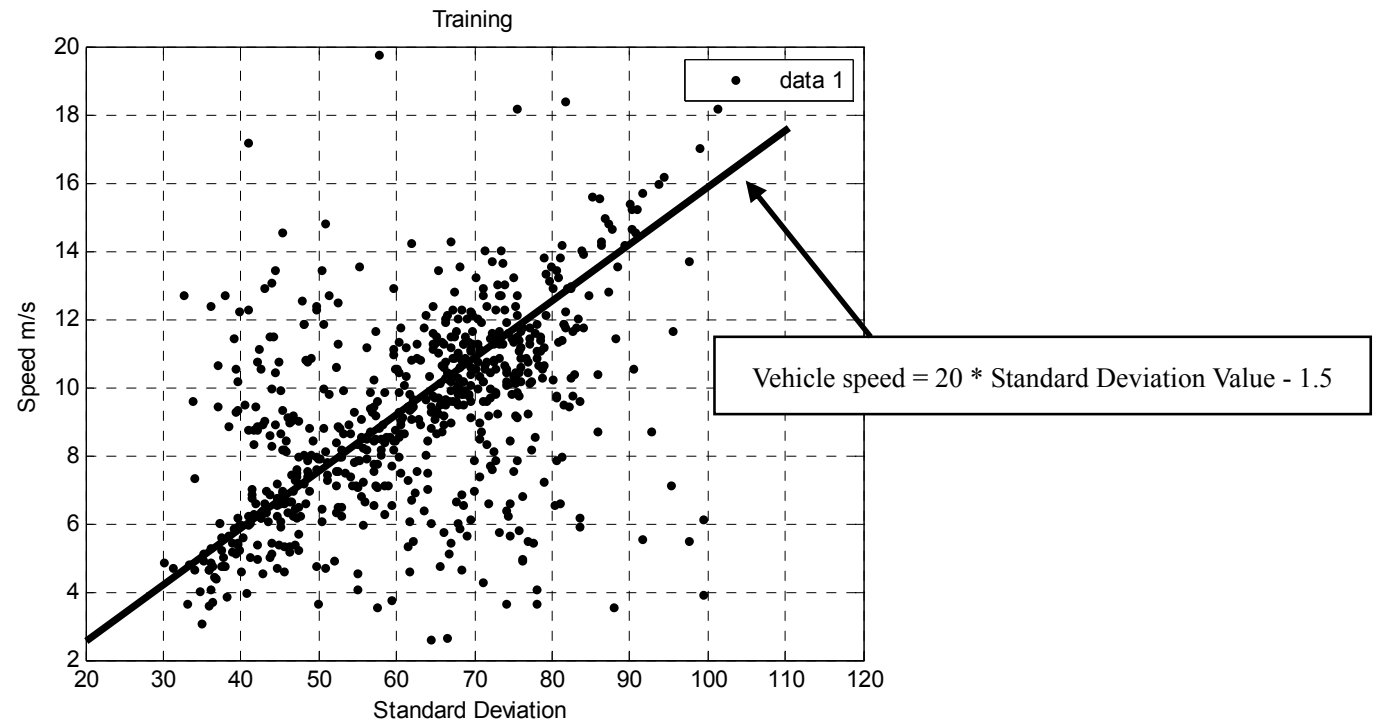

Figure 12. Library of 700 samples for Standard deviation Vs speed of vehicle 


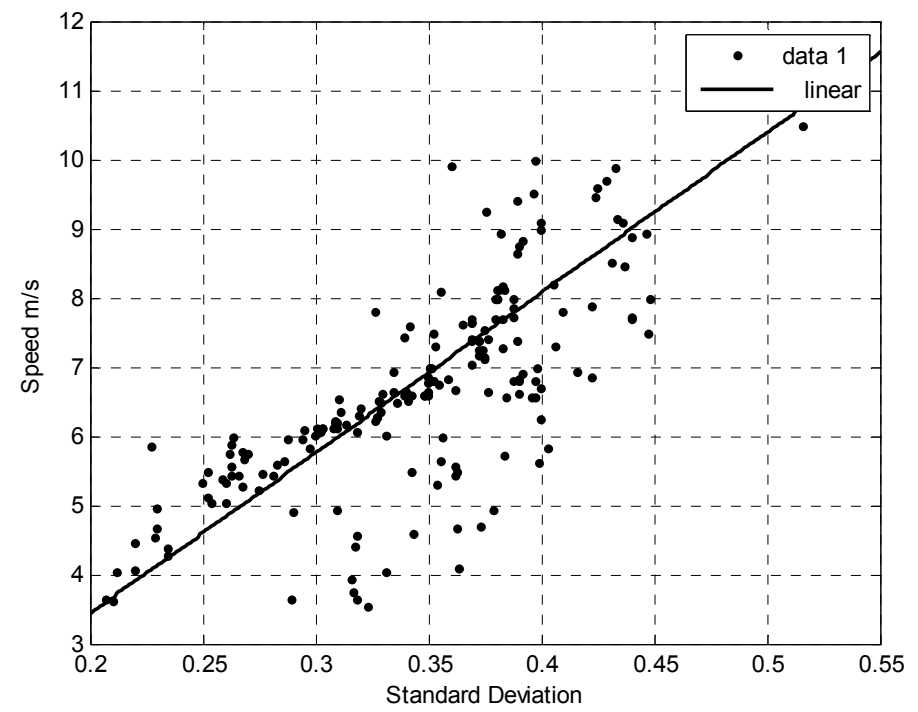

Figure 13. Testing of 150 samples Standard deviation Vs speed of vehicle

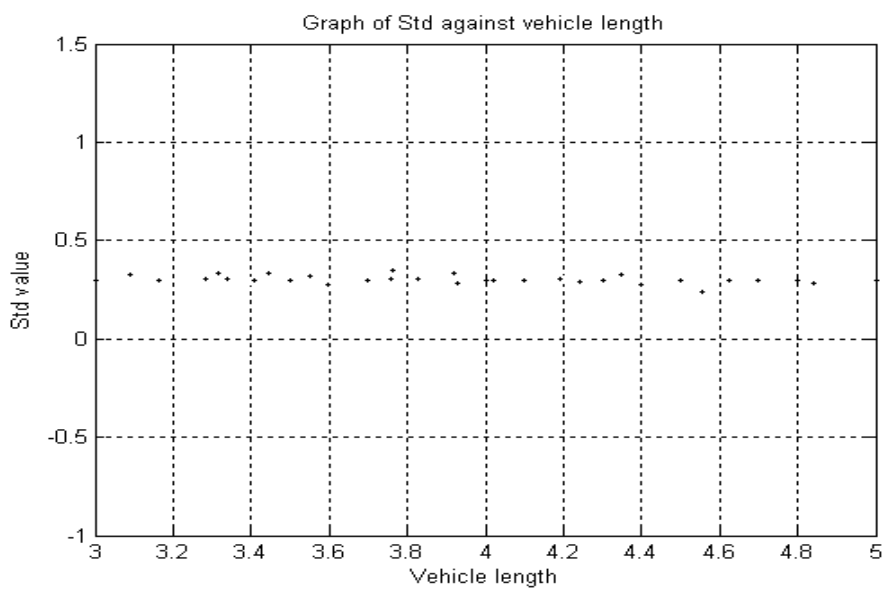

Figure 14. Vehicle length Vs Standard deviation 